\title{
Development of A Novel Virtual Coordinate Measuring Machine
}

\author{
Yang $\mathrm{Hu}^{*}$, Qingping Yang, Peng Wei \\ School of Engineering and Design \\ Brunel University \\ Middlesex UB8 3PH, UK \\ *yang.hu@brunel.ac.uk
}

\begin{abstract}
Existing VCMMs (Virtual Coordinate Measuring Machine) have been mainly developed to either simulate the measurement process hence enabling the off-line programming, or to perform error analysis and uncertainty evaluation. Their capability and performance could be greatly improved if there is a complete solution to cover the whole process and provide an integrated environment. The aim of this study is to develop such a VCMM that not only supports measurement process simulation, but also performs uncertainty evaluation. It makes use of virtual reality techniques to provide an accurate model of a physical CMM, together with uncertainty evaluation. An interface is also provided to communicate with CMM controller, allowing the measuring programs generated and simulated in the VCMM to be executed or tested on the physical CMM afterwards. This paper discusses the proposal of a novel VCMM design and the preliminary results.
\end{abstract}

Keywords- VCMM (Virtual Coordinate Measuring Machine); virtual measurement; metrology; evaluation of measurement uncertainty

\section{INTRODUCTION}

Today CMMs are widely used in manufacturing industry to measure three-dimensional physical components. There have been several virtual CMMs developed over the recent years [1, 2, 3, 4]. Typically the existing VCMMs fall into two categories. The first kind of VCMM is the simulation of the actual measurement process and in particular, the path planning of a physical CMM. It focuses on representing the inspection path planning methodology in the virtual environment, which enables the off-line programming of the CMM concerned. As a virtual CMM, it also facilitates the low-cost, accident-free training/learning of complicated CMM operations. Several researchers have proposed very interesting approaches and introduced some new techniques into the development of VCMM, for example, the HVCMM (Haptic Virtual Coordinate Measuring Machine) $[1,5,6]$. It allows the user to control the virtual probe by moving a joystick, and also makes use of the haptic technology to provide a force feedback when collision happens between the VCMM and a workpiece. This gives the operator a simulated sense very similar to the operating of a real CMM $[1,5,6]$. Another research has shown that the efficiency of simulation can be improved by better user interface and proper user guidance [7].

The second kind of VCMM is basically a software tool intended for the evaluation of measurement uncertainty. This kind of VCMM is essentially a mathematical model of the CMM measurement process. It takes into account various uncertainty sources, and based on the knowledge of the influence quantities, these sources of uncertainties can be combined together to generate the expanded uncertainty and also give the distribution of possible results. Many models and methods have been studied and developed, such as Monte Carlo methods [2], Kinematic analysis [8], and error compensation model etc. [3, 9]. Further more, a group of researchers from NEDO VCMM team have been trying to boost the international standardization of development of VCMM [4].

However, none of these current VCMMs provide an integrated solution, i.e. allowing user to simulate the CMM operations in a virtual environment (off-line programming by operating virtual probes), then calculate the desired geometric features based on the coordinates of a set of points collected from the virtual measurement just like CMM console software does, whilst at the same time it is also able to evaluate the measurement uncertainty. This research aims to develop a complete and accurate model of the CMM, which allows users to carry out, in the virtual system, every operation that they would expect on a physical CMM, with additional capability of uncertainty evaluation. In addition, if desired, the user can also export the developed measurement program to a physical CMM and run it in a real CMM. This approach will greatly facilitate fast and cost effective off-line path-planning and uncertainty evaluation. In this paper, the basic design of this novel VCMM is proposed, and the preliminary results are reported and discussed.

\section{PROPOSED VCMM SYSTEM}

The proposed VCMM system consists of four main parts: a virtual CMM model, a Movement Control and Contact Detection Module, a Result Processing/Calculation Module, and an Uncertainty Evaluation Module. 
The virtual CMM model, as shown in Figure 1, is built using VRML language. The VRML standard provides good interactivity and compatibility (especially for web distribution). In the program, when a virtual world is created from this VRML model, it accepts user input from a joystick (standard input devices like keyboards and mice are also supported). Users carry out the path planning by using the joystick to move the virtual probe, and define the measurement points just like operating a real CMM. The virtual probe can be driven in the same manner as on a physical CMM machine, e.g. with a high moving speed and a lower measurement speed.

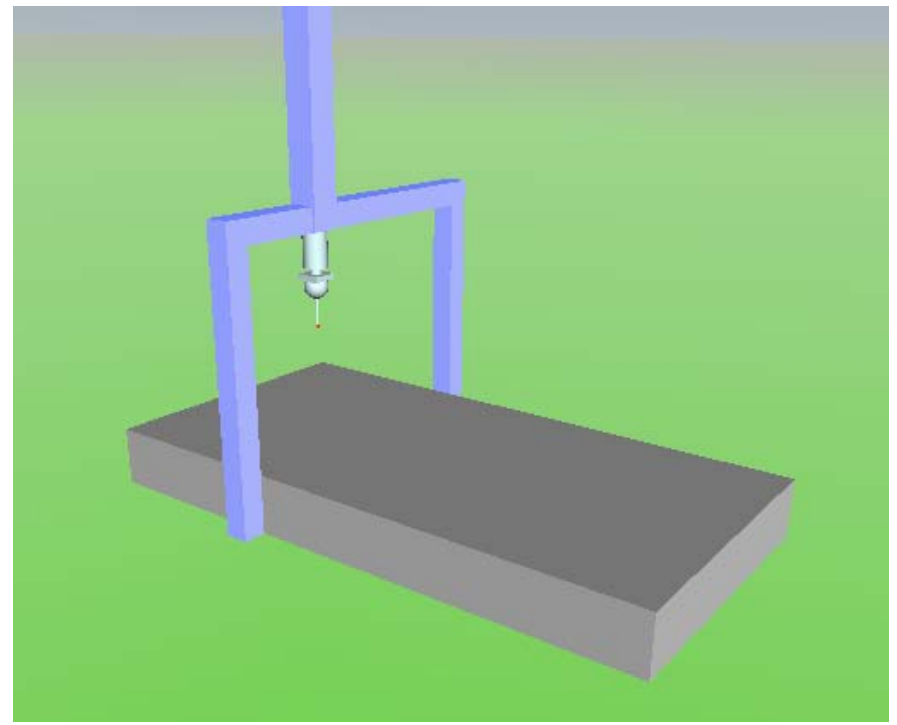

Figure 1. VRML Model of CMM

When the probe moves, the Movement Control and Contact Detection Module monitors the change of probe position and checks the position against the workpiece surface (and other potentially contactable objects, such as the CMM measuring table). When the virtual probe touches the surface of the virtual workpiece, a very small overlap occurs between the stylus-tip and workpiece surface. This overlap, which is equivalent to the effect of overtravel in real CMM, is detected by the Contact Detection module and is subsequently calculated to determine the correct measured position on the workpiece. The coordinates of the measured position are recorded for later processing. At the same time, as a system output control, the Movement Control and Contact Detection Module beeps and stops the probe from moving further into the workpiece (Figure 2 ). The probe then bounces back to a previous position - a small distance away from the workpiece surface, to be ready for next movement.

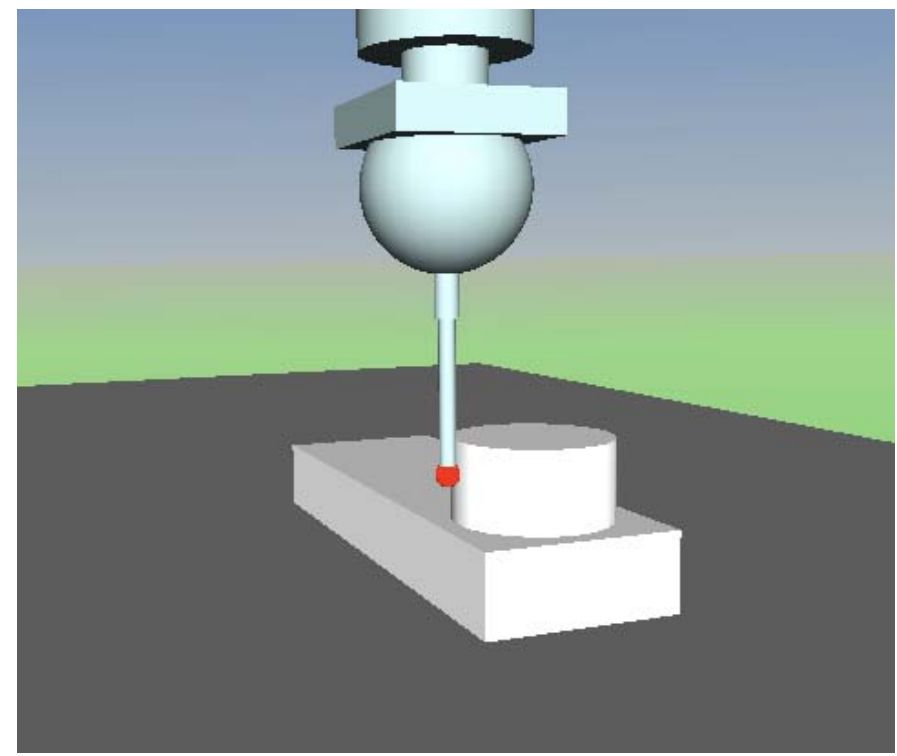

Figure 2. Probe Contacting Workpiece

Figure 3 outlines the VCMM flow chart. Just like a CMM does not directly measure geometric features such as distances, diameters or angles, the VCMM gathers a set of points generated in the virtual measurement and passes them on to the Result Processing/Calculation Module, where desired geometric features are calculated.

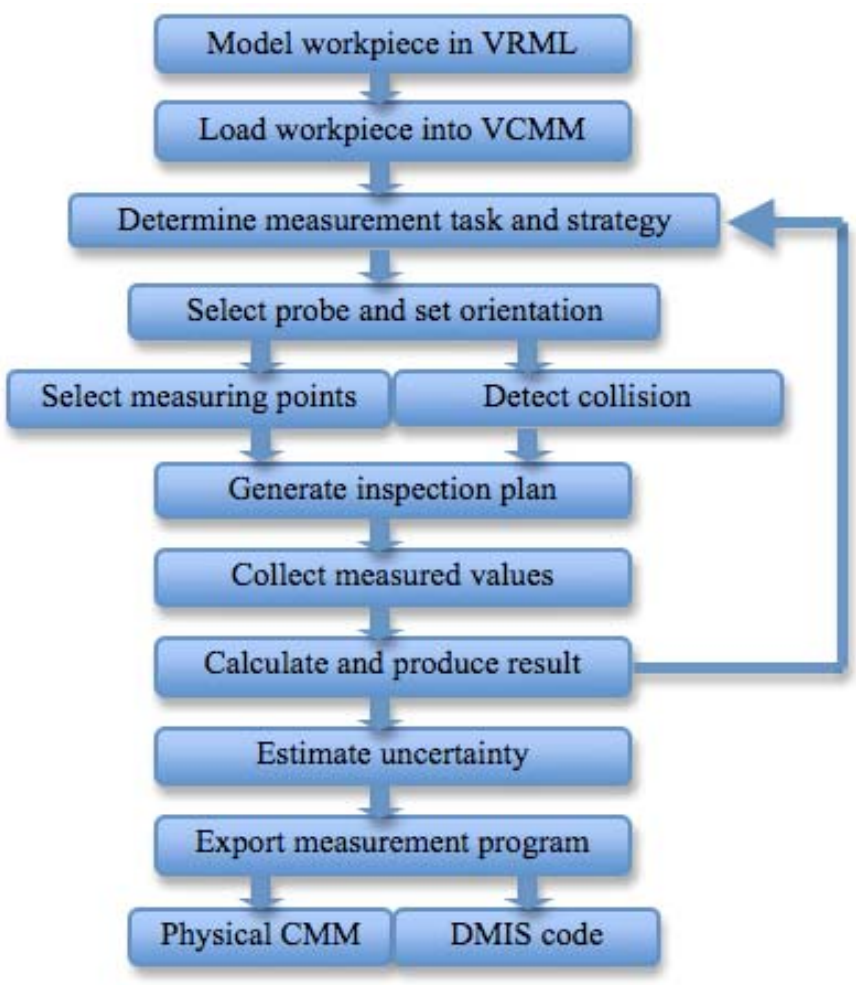

Figure 3. Working Flow Chart of the VCMM

After least squares fitting and other calculations in the Result Processing/Calculation Module, the results are produced and then output to Uncertainty Evaluation Module. This 
module has not been completed yet. A novel Uncertainty Evaluation Module is being developed for virtual measurement based upon the existing approaches.

Alternatively, as shown in figure 4 , the points gathered in the virtual measurement can also be passed to the calculation software of a specific CMM through its interface. This way the uncertainty produced by the data processing system of a CMM can also be included and evaluated in the later stage.

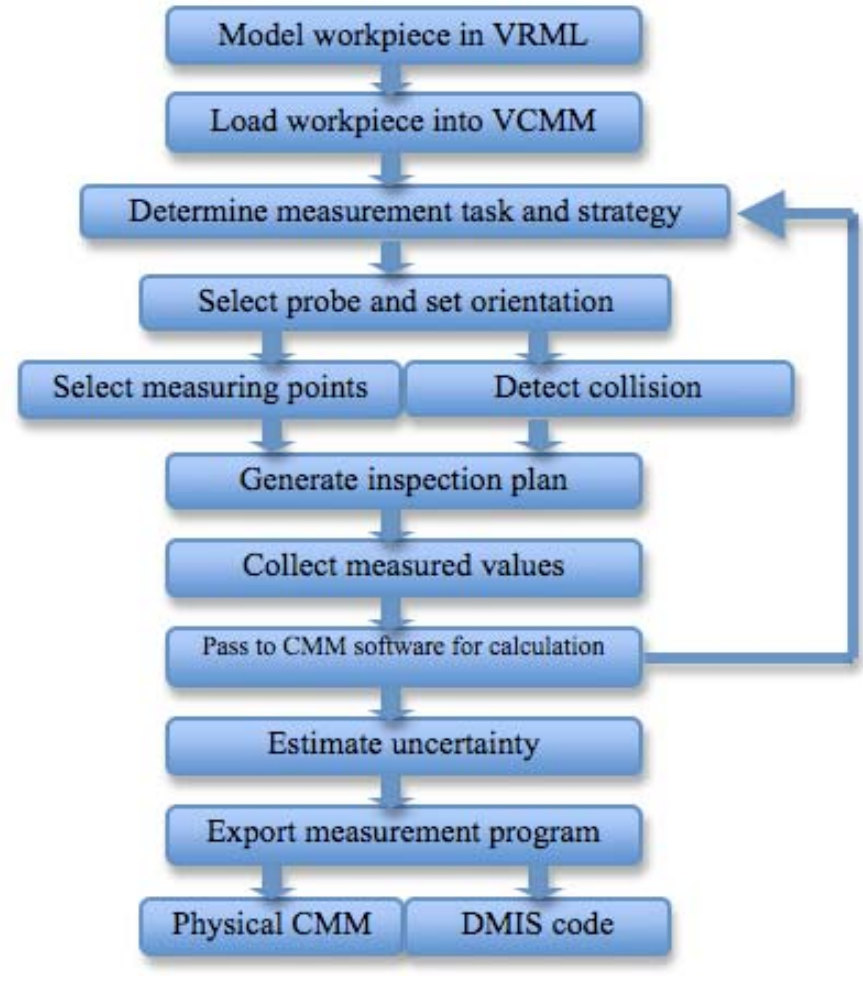

Figure 4. Flow Chart of VCMM, with CMM software calculating results

In addition, VCMM users can review, change and optimise the measurement programs in terms of measurement speed and/or uncertainty. Finally the program can be either exported as DMIS Codes or transferred automatically via an interface to a physical CMM console and run in its physical environment.

\section{PROGRESS OF DEVELOPMENT}

The 3D model of the CMM has been built as a generic CMM in terms of range and capacity and can be easily modified to simulate a specific model. It makes use of the inner coordinate system of VRML virtual world, which is flexible and transformable, to provide a typical three-dimensional coordinate system for the motion of the three orthogonal axes of the VCMM. The position of each axis is controlled by retrieving and modifying the translation fields of each axis node.

The Movement Control and Contact Detection Module has been developed using MATLAB. A 'vcmm' class is built to represent the virtual machine, and the Movement Control and Contact Detection Module is two of its methods (functions). The 'vcmm' class has properties like 'probe position' 'contact_number' 'contact_record' etc. to store data like the current coordinates of the probe, the ordinal number of contacts and the records of each measured point. It also has a 'cmm world' property, which stores the handle to the virtual world of VCMM. When program starts, one of the GUI callback initializes the VCMM by making an instance of 'vcmm' class. This initialization creates a virtual world from the VRML model, initializes properties for storing data, and maps the coordinates in the 'vcmm' object to the virtual world, and then draws the scene. Workpiece can be dynamically loaded into the virtual world by creating an instance of 'workpiece' class, which adds nodes forming the workpiece to the virtual world and provides handles to these nodes.

When user tries to move the probe via the interface, the inputs from joystick or keyboard/mouse are passed to method move probe(), and may be used to update the virtual world by synchronizing the coordinates in the object to the virtual world to drive the probe. However before actually updating the virtual world with the new probe position, the contact_detection() function is called to determine whether a contact occurs. In typical CMM contexts, when measuring a workpiece, the moving parts are three axes including vertical spindle and the probe system. In this VCMM, only the vertical spindle and the probe are considered to be possible to contact other objects. As for the static parts, those considered as 'contactable' are the measuring table surface and, of course, the workpiece when it is loaded (workpiece fixers are planned to be added in the future). All these potentially contactable objects can be broken down to VRML geometric primitives. To detect contact, two structure arrays, moving parts and static parts, are maintained to store geometrical information about all these primitives, one for moving parts and one for static parts. These two arrays are built when the VCMM initializes, and can be dynamically updated when probe moves, or be rebuilt when workpiece loads or changes. The data stored in the arrays include shape, translation (for calculating the absolute position), rotation (orientation) and size, and are used to find overlap with each other. After a measurement starts, in each category of moving_parts and static_parts, the geometric primitives that make up objects in the same category are fixed in reference to each other, and are not tested for contact with each other. Whenever the position of probe changes, contact detection() updates the moving parts array, and tests each element in moving_parts against all elements in static parts. Once an overlap is found, meaning a contact occurs, contact detection() records the current position of probe (which can be then calculated to generate corrected contact point), and returns a flag indicating that a contact is made. The move probe() function then responds to the result: stops the probe (or rarely the vertical spindle) from moving further into the workpiece or table, bounces back the stylus a little bit, and fires an event to the interface for beeping and other outputs. After a measurement completes, the array contact record storing all the records of measured pointes are passed to Result Processing/Calculation Module for further process.

The Result Processing/Calculation Module is developed to implement the functionality of typical CMM measurement program. When a specific measurement begins, the user needs to choose a geometrical feature to measure (e.g. line, circle, 
sphere, etc.). This choice is marked in a variable. Once the measurement ends, the records of measured points contact record together with the choice of feature are passed to Result Processing/Calculation Module. This module calls the corresponding functions according to the kind of chosen feature, to carry out certain type of curve fitting or surface fitting, using least squares method. The results can be represented as either the dimensions of the geometrical feature or the coefficients of its equation. The deviations of the measured points from the least square fit are also computed and output for further reference and process. These results are then passed to the Uncertainty Evaluation Module, which is still under development, for further analysis.

Like mentioned before, alternatively the Result Processing/Calculation Module can be replaced with the calculation software of the real CMM by exporting measured points to it. As algorithms, implementation and configurations differ on different CMM systems, this allows for the uncertainty of the calculation software of a specific CMM system to be considered and estimated in the Uncertainty Evaluation Module.

The current state of this VCMM can be used as a fully functional off-line path planning tool and a great teaching platform for training CMM operators, since it can simulate the whole procedure of CMM operations. The effort of future work will be focused on the integration with the Uncertainty Evaluation Module, and the development of the interface to CMM console.

\section{CONCLUSIONS}

This paper presents a novel VCMM system, which makes use of both 3D VR and uncertainty evaluation methodology, to provide an integrated environment for simulating CMM operations and carrying out virtual uncertainty evaluation. It has a user friendly interface in an operation manner similar to physical CMMs, which make this system easy to use and suitable for CMM operation training. This system not only enables off-line programming and uncertainty evaluation, but also speeds them up in a cost effective way. The planned interface to CMM console makes this system virtually a control centre, and all the measurement tasks can be programmed, tested and evaluated prior to running in physical environment. The adoption of VRML modeling technology, which is a widely supported standard, offers good extensibility and makes it easier to distribute the system, even possibly as a web service. This research has shown great potential of an integrated VCMM system.

\section{References}

[1] Y. H. Chen, Y. Z. Wang, Z. Y. Yang, "Towards a haptic virtual coordinate measuring machine," International Journal of Machine Tools \& Manufacture, vol. 44, pp. 1009-1017, 2004.

[2] B. van Dorp, H. Haitjema, F. Delbressine, R. Bergmans, P. Schellekens, "Virtual CMM using Monte Carlo methods based on frequency content of the error signal," Proceedings of SPIE - The International Society for Optical Engineering, vol. 4401, pp. 158-167, 2001.

[3] H. J. Pahk, M. Burdekin, G. N. Peggs, "Development of virtual coordinate measuring machines incorporating probe errors," Proceedings of the Institution of Mechanical Engineers, Part B: Journal of Engineering Manufacture, vol. 212, no. B7, pp. 533-548, 1998.

[4] K. Takamasu, R. Furutani, T. Kurosawa, T. Takatsuji, F. Wäldele, H. Schwenke, N. Brown, E. Jaatinen, "International standard development of virtual CMM (coordinate measuring machine)," Report of NEDO VCMM Team, available from: <http://www.nedo.go.jp/itd/grante/report/99pdf/is-01e.pdf> [6 October 2008], April 1999 - March 2002.

[5] Z. Yang, Y. Chen, "Inspection path generation in haptic virtual CMM," Computer-Aided Design \& Applications, vol. 2, no. 1-4, pp. 273-282, 2005.

[6] Y. H. Chen, Z. Y. Yang and Y. Z. Wang, "Haptic modeling for a virtual coordinate measuring machine," International Journal of Production Research, vol. 43, no. 9, pp. 1861-1878, 1 May 2005.

[7] N. C. Junior, A. J. Abackerli, L. A. Consularo, "User interface design for VCMMs: an approach to increase fidelity and usability," Proceedings of 2006 IEEE International Conference on Virtual Environments, HumanComputer Interfaces and Measurement Systems, La Coruña, Spain, 1012 July 2006, pp. 130-135.

[8] D. Liu, X. Chen, S. Wang, R. Wei, "Kinematic analysis and simulation for a parallel - link coordinate measuring machine based on ADAMS environment," Modular Machine Tool \& Automatic Manufacturing Technique, no. 11, pp. 18-21, 2007.

[9] Q. Yang, C. Butler, P. Baird, "Error compensation of touch trigger probes," Measurement: Journal of the International Measurement Confederation, vol. 18, no. 1, pp. 47-57, May 1996. 